\title{
Malformação arteriovenosa dural do seio transverso-sigmóide causando neuralgia trigeminal
}

\author{
César de Paula Lucas, Válter da Costa, Joaquim da Costa, Luís Fernando Martins, \\ Sebastião E. Melo-Souza, César N. Raffin
}

Departamento de Neurologia e Neurocirurgia do Instituto de Neurologia de Goiânia, Goiás

\section{RESUMO}

Os autores relatam um caso raro de malformação arteriovenosa dural (MAVD) do seio transverso-sigmóide esquerdo, causando neuralgia do trigêmeo. O paciente foi submetido a cirurgia para esqueletização do seio transversosigmóide com ressecção dos dois terços distais do seio transverso e dos dois terços proximais do seio sigmóide. O procedimento cirúrgico foi suficiente para proporcionar a cura angiográfica e clínica, com um seguimento de oito meses. Foi realizada uma completa revisão bibliográfica encontrando-se apenas cinco casos descritos de pacientes com MAVD e neuralgia trigeminal.

\section{PALAVRAS-CHAVE}

Malformação arteriovenosa dural. Neuralgia do trigêmeo. Seio transversosigmóide.

\section{ABSTRACT}

Transverse-sigmoid sinus dural arteriovenous malformation presenting as trigeminal neuralgia. Case report

The authors report a rare case of transverse-sigmoid dural arteriovenous malformation (DAVM) presenting as trigeminal neuralgia in the distribution of left V3. The patient was treated surgically by the sinus skeletonization technique with the resection of the distal $2 / 3$ of the transverse sinus and the proximal $2 / 3$ of the sigmoid sinus. Clinical and angiographic cure was achieved in our patient with a folow-up of 8 months. Furthermore, a thorough review of the English language literature was carried out, compiling only five cases of trigeminal neuralgia due to DAVM.

\section{KEY WORDS}

Dural arteriovenous malformation. Transverse-sigmoid sinus. Trigeminal neuralgia.

\section{Introdução}

As malformações arteriovenosas durais (MAVD) representam entre $10 \%$ e $15 \%$ das malformações arteriovenosas intracranianas gerais ${ }^{3,4,8}$.

As neuralgias trigeminais secundárias respondem por apenas $2 \%$ dos casos de neuralgia trigeminal geral ${ }^{6}$ e estão, na maioria das vezes, relacionadas a lesão expansiva na região da cisterna trigeminal. 
As MAVD podem apresentar-se clinicamente por uma plêiade de sinais e sintomas dependentes da sua aferência, eferência, patência e localização ${ }^{9}$. Os sinais e sintomas usuais variam desde cefaléia inespecífica, tinnitus pulsátil, quemose, dor ocular, exoftalmia pulsátil, ataxias, déficits medulares e neurológicos progressivos até a hemorragias fatais intraparenquimatosas ou subaracnóideas ${ }^{7}$. No entanto, MAVD levando a neuralgia trigeminal é considerada uma manifestação clínica rara $25,6,10,11$.

Apresentamos um caso clínico incomum de MAVD do seio transverso-sigmóide exibindo neuralgia trigeminal típica. A MAVD foi tratada eficientemente através da esqueletização e remoção do seio transversosigmóide acometido.

\section{Relato do caso}

V.L.Q., 50 anos de idade, sexo masculino, cor branca, procedente de Goiânia. Há cerca de seis anos passou a perceber, progressivamente, dor em choque, lancinante no território do terceiro ramo trigeminal esquerdo, a qual vinha respondendo à carbamazepina prescrita por neurologista de outro serviço. Apresentava ainda tinnitus pulsátil no ouvido esquerdo que se assemelhava a uma "cachoeira". Nos últimos dez meses, os episódios dolorosos recrudesceram apesar da carbamazepina em altas doses. Foi encaminhado ao Departamento de Dor do Instituto de Neurologia de Goiânia (ING) para ser submetido a termocoagulação do gânglio de Gasser.

O estudo de tomografia computadorizada do crânio revelou dilatações venosas na região temporoccipital esquerda. Em virtude desse achado, o paciente foi submetido a estudo angiográfico que revelou uma grande MAVD do seio transverso-sigmóide esquerdo, nutrida pelo ramo posterior da artéria meníngea média e ramo muscular da artéria vertebral; havia trombose parcial do seio sigmóide e drenagem venosa retrógrada ao sistema de Labbé, seio petroso superior e veias leptomeníngeas esquerdas (Figs. 1 e 2). O paciente foi submetido a uma craniotomia (CPL e VC) supra e infratentorial, com ampla exposição da junção transverso-sigmóide esquerda, seguida de sua completa esqueletização. Por fim, ressecou-se os dois terços distais do seio transverso esquerdo e os dois terços proximais do seio sigmóide esquerdo, juntos com a MAVD (Fig. 3). Controle angiográfico, realizado 60 dias após o ato cirúrgico, revelou completo desaparecimento da MAVD (Figs. 4 e 5). Desapareceram também a dor trigeminal e o tinnitus pulsátil. Não foi registrado nenhum déficit neurológico no pós-operatório.

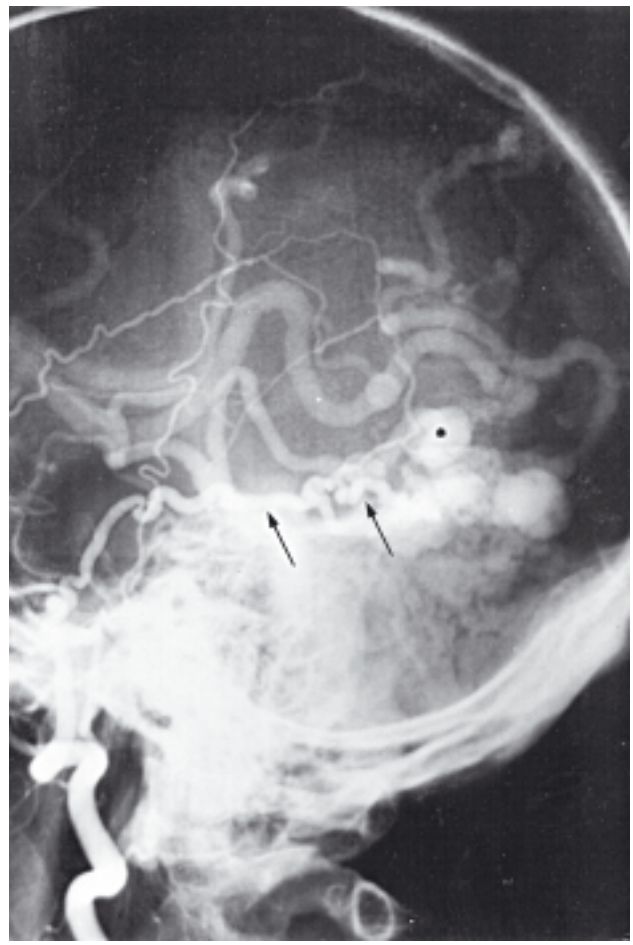

Figura 1 - Angiografia carotídea externa esquerda em perfil. Note o ramo posterior da artéria meníngea média esquerda (setas) nutrindo a MAVD dural do seio transverso-sigmóide esquerdo parcialmente trombosado, dilatação venosa aneurismática displásica (asterisco) e a drenagem venosa leptomeníngea.

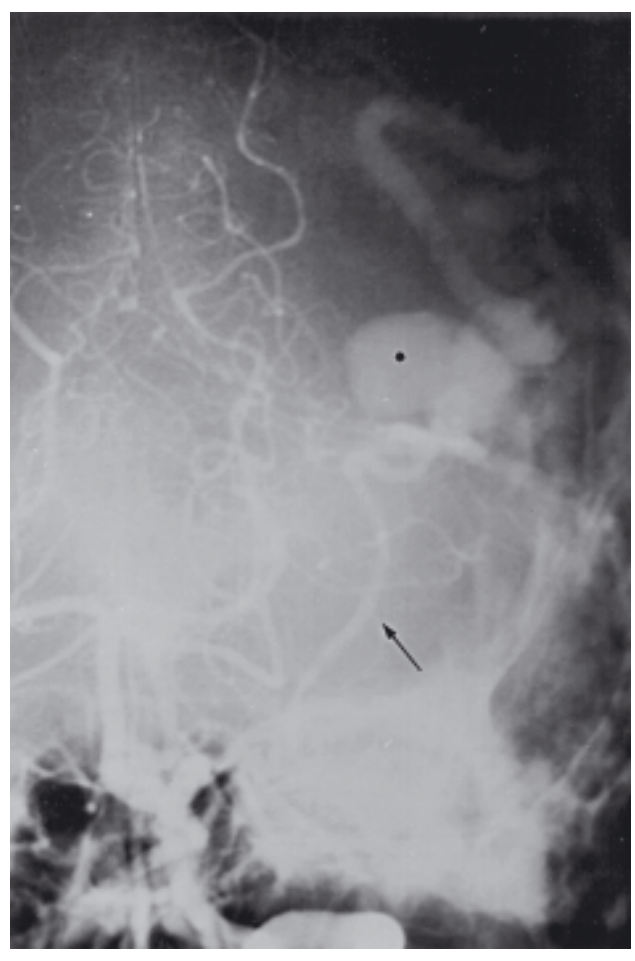

Figura 2 - Angiografia da artéria vertebral esquerda. Presença de ramo muscular (seta) nutrindo a MAVD com a dilatação venosa aneurismática displásica (asterisco). 


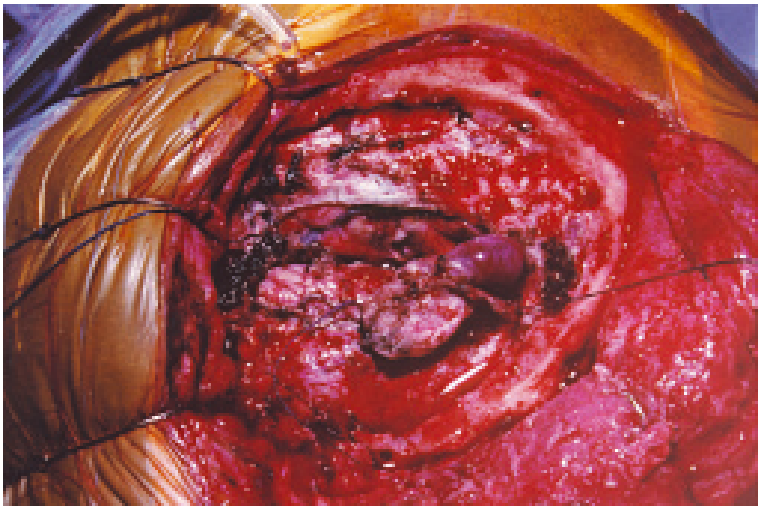

Figura 3 - Esqueletização do seio transverso-sigmóide esquerdo com ressecção da MAVD e da dilatação venosa aneurismática displásica, por meio de carniotomia supra e infratentorial.

\section{Discussão}

A associação entre MAVD e neuralgia do trigêmeo é extremamente rara. A revisão bibliográfica, realizada através da Medline e lista de referências dos artigos examinados, revelou apenas cinco casos em toda literatura ${ }^{2,5,6,10,11}$.

O paciente em questão apresentava tinnitus pulsátil associado a hipoestesia e neuralgia trigeminal do terceiro ramo esquerdo há cerca de seis anos. $\mathrm{O}$ tinnitus pulsátil é a manifestação clínica mais freqüente nas $\mathrm{MAVD}^{7}$. O tinnitus, lembrando cascata ou cachoeira, irradiando ao ouvido ipsilateral à MAVD é decorrente do alto fluxo turbilhonado existente no interior da fístula. A neuralgia do trigêmeo, nesses casos, é conseqüente à trombose venosa sinusal e hipertensão venosa ${ }^{8,9}$. A trombose venosa sinusal é usualmente o mecanismo de disparo para aquisição da MAVD. A recanalização do seio acometido costuma ser a regra. Entretanto, alguns seios venosos mantêm o mecanismo inicial de trombose, redirecionando o fluxo venoso de drenagem para seios venosos profundos e veias leptomeníngeas, desencadeando um elevado grau de hipertensão venosa nesses sistemas, proporcionando o aparecimento de displasias venosas tendendo a dilatações venosas aneurismáticas. No caso apresentado, a trombose parcial do seio sigmóide redirecionou retrogradamente o fluxo sangüíneo de drenagem tanto para o sistema de Labbé quanto ao seio petroso superior e veia petrosa (Figs. 1 e 2) com compressão no nível da zona de entrada do nervo trigêmeo.

Ao estudo angiográfico, o paciente apresentava drenagem venosa leptomeníngea à região temporoccipital esquerda, portanto um padrão do tipo IV de Cognard $^{4}$ (Figs. 1, 2 e 3).

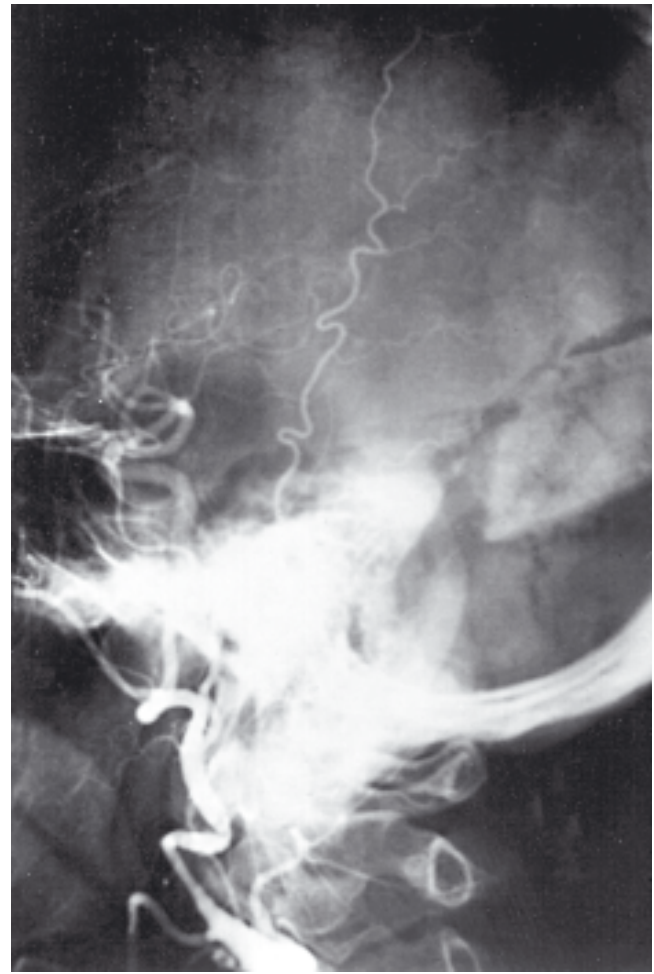

Figura 4 - Angiografia carotídea externa esquerda, no pós-operatório, revelando a completa ressecção da MAVD dural do seio transverso-sigmóide.

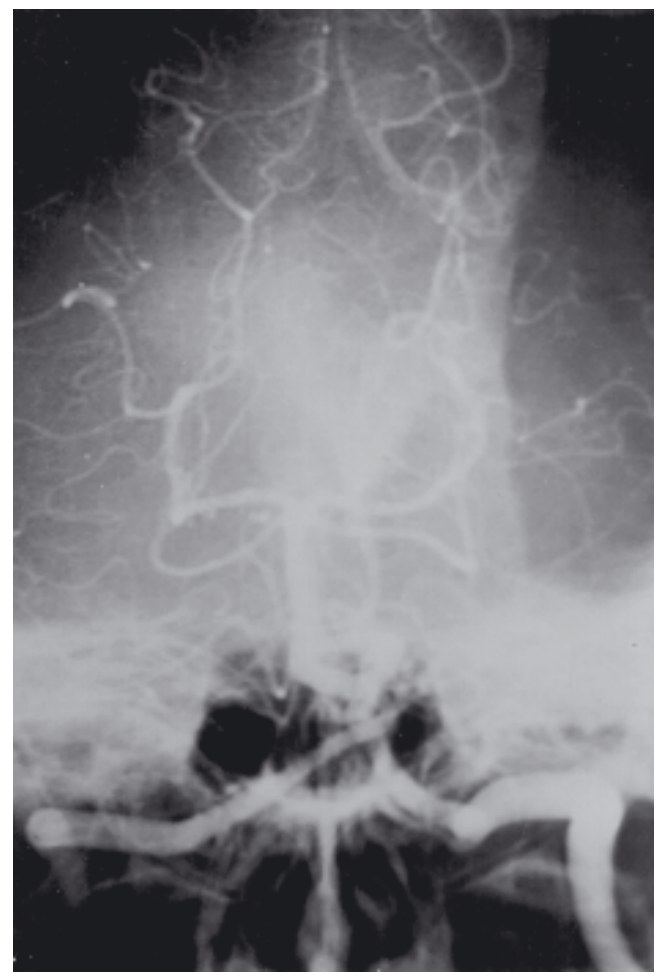

Figura 5 - Angiografia da artéria vertebral esquerda, no pós-operatório, confirmando a cura angiográfica do caso. 
Awad e cols. ${ }^{1}$ associaram estatisticamente o risco de hemorragia com a presença de drenagem venosa leptomeníngea. Em virtude disso, indicamos a cirurgia para esqueletização dos seios transverso e sigmóide.

Harders e cols. ${ }^{5}$ apresentaram o caso de um paciente com 53 anos de idade, sofrendo de neuralgia trigeminal típica por cinco anos. Estudo angiográfico revelou tratar-se de MAVD do seio transversosigmóide. Após a falência da terceira intervenção cirúrgica para microdescompressão neurovascular, optaram pelo isolamento dos seios venosos da duramáter com eliminação da MAVD e da neuralgia.

Borden e cols. ${ }^{2}$ publicaram um caso de MAVD no seio petroso superior, cursando com dor facial. Esse paciente de 39 anos foi eficientemente tratado com a excisão do nidus dural e interrupção da veia de drenagem.

Ito e cols. ${ }^{6}$ apresentaram o caso de um paciente de 65 anos, com neuralgia do trigêmeo cujas termocoagulações sucessivas do gânglio de Gasser não foram suficientes para interromper a sintomatologia do paciente. Nova tentativa, desta feita por embolização endoarterial, foi suficiente para garantir o controle da dor por apenas dois meses, recorrendo a seguir. Uma segunda sessão de embolização foi realizada, de modo combinado com uma cirurgia, a fim de se interromper com clipe de Sugita a veia de drenagem da MAVD. Não houve recidiva do quadro álgico do paciente.

Nosso procedimento cirúrgico consistiu de uma esqueletização dos dois terços distais do seio transverso e dos dois terços proximais do seio sigmóide, seguido de ressecção em bloco da MAVD e seio transverso-sigmóide acometidos. A cura angiográfica foi demonstrada eficientemente no controle radiológico após dois meses (Figs. 4 e 5) e, a cura clínica, por meio seguimento durante oito meses.

Consideramos a esqueletização sem ou com remoção do seio acometido, precedida ou não de embolização, condição fundamental para a chamada "cura angiográfica e clínica" desses pacientes portadores de MAVD.

\section{Referências}

1. AWAD IA: Intracranial dural arteriovenous malformations: factors predisposing to an aggressive neurological course. J Neurosurg 72:839-850, 1990.

2. BORDEN JA, WU JK, SHUCART WA: A proposed classification for spinal and cranial dural arteriovenous fistulous malformations and implications for treatment. J Neurosurg 82:166-179, 1995.

3. BROWN RD Jr, WIEBERS DO, NICHOLS DA: Intracranial dural arteriovenous fistulae: angiographic predictors of intracranial hemorrhage and clinical outcome in nonsurgical patients. J Neurosurg 81:531-538, 1994.

4. COGNARD C, GOBIN YP, PIEROT L, BAILLY AL, HOUDART E, CASASCO A, CHIRAS J, MERLAND JJ: Cerebral dural arteriovenous fistulas: clinical and angiographic correlation with a revised classification of venous drainage. Radiology 194:671-680, 1995.

5. HARDERS A, GILSBACH J, HASSLER W: Dural AV malformation of the lateral and sigmoid sinuses as possible cause of trigeminal neuralgia. Case report. Acta Neurochirur (Wien) 66:103-107, 1982.

6. ITO M, SONOKAWA T, MISHINA H, SATO K: Dural arteriovenous malformation manifesting as tic douloureux. Surg Neurol 45:370-375, 1996.

7. LUCAS CP: Malformação arteriovenosa dural intracraniana - MAVD. Neurocirurgia Contemporânea Brasileira 2: 1-5, 1997

8. LUCAS CP, OLIVEIRA E, TEDESCHI H, SIQUEIRA M. LOURENZI M, PISKE RL, CONTI M, PEACE D: Sinus skeletonization: a treatment for dural arteriovenous malformations of the tentorial apex. Report of two cases. J Neurosurg 84:514-517, 1996.

9. LUCAS CP, ZABRAMSKI JM, SPETZLER RF, JACOBOWITZ R: Treatment for intracranial dural arteriovenous malformations: a meta-analysis from the english language literature. Neurosurgery 40:1119-1132, 1997.

10. MENDELOWITSCH A, RADUE EW, GRATZL O: Aneurysm, arteriovenous malformation and arteriovenous fistula in posterior fossa compression syndrome. Eur Neurol 30:338-342, 1990.

11. OTT D, BIEN S, KRASZNAI L: Embolization of a tentorial dural arterio-venous fistula presenting as atypical trigeminal neuralgia. Case report and review. Headache 33:503-508, 1993.

Original recebido em março de 1998

Aceito para publicação em outubro de 1998

Endereço para correspondência:

César de Paula Lucas

Instituto de Neurologia de Goiânia

Praça T-18, 140 Setor Bueno

CEP 74210250 - Goiânia, GO 